\title{
175. Pursuit Motion in Patients with Head and Neck Injuries
}

\author{
Yoshinao Hukado, Masayasu Hayashi, Mitsuko NagaI \\ and Takashi Sakimoto \\ Department of Ophthalmology, Kanto-Rosai Hospital
}

The smooth pursuit motion of the eye which was elicited by moving target was recorded by means of electrooculogram (EOG). The subjects which were selected for this procedure were 105 cases with the head and the neck injuries. All cases did not show any impairment of the visual acuity and the visual field. The age ranged from 6 to 67 years old. The results were evaluated from EOG data:

1. Irregularities of the pattern due to smooth pursuit motion of the eyes were major results in the present study.

2. 28 cases out of 105 revealed the severe disturbance of pursuit eye movements.

3. The saccadic motion, on the other hand, were assumed to be almost normal in the present cases.

4. Unilateral impairment of the eye was not obtained.

Until now, in spite of various and broad complaints, there were no clinical tests for evaluating or finding out the positive impairments in those patients, however, EOG test, especially, smooth pursuit moion seemed to be affected. This is the major point to emphasize in the present results.

\section{Nystagmus and Optokinetic Nystagmus in the Patient with Whiplash Injury}

\author{
Hsu Jui Kuang and Tuneo OHNo \\ Neurosurgical Department, Kanto Rosai Hospital \\ Katumi SUzUKI and Nobuyuki TUZUKI \\ Orthopaedic Department, Kanto Rosai Hospital
}

We reported on the result of nystagmus and optokinetic nystagmus in 194 cases of so called whiplash injury. All cases neglected head injury. Nystagmus is observed in 70 cases i.e. 36 per cent, but other neurological findings are negative in the almost all cases except for equilibratory, cervical radiculopathic, and sensory disturbances. O.K.P. test is a examination for the oculomotor function and the 